\title{
COUGH VIEWED BRONCHOLOGICALLY
}

\author{
Louis H. Clerf, M. D. \\ Philadelphia, Pa.
}

Cough is a common symptom which often is not properly evaluated nor effectively treated. The physician usually is confronted with the questions: what is the cause of the cough; what should be done about it? While it may be distressing and purposeless it more often is a necessary and useful act so that suppression would be hazardous. Cough can be voluntarily produced but it commonly is a purely reflex response and is often reinforced by volitional effort.

Cough is dependant upon a local stimulus which originates nerve impulses that are transmitted by afferent nerves'to the medulla. The reflex arc is completed through motor or efferent fibers innervating the respiratory and laryngeal muscles. The source of the impulses commonly originates in the air passages and stimulates sensory nerve endings of the pneumogastric and glossopharyngeal nerves. These impulses are carried to the respiratory centres in the medulla and excite and coordinate the spinal centers. There result increased activity of the inspiratory centre, stimulation of the adductor center of the larynx and increased excitation of the expiratory center. The act of coughing therefore can be divided into three phases namely, inspiratory, compressive and expiratory. During the inspiratory phase there is a deep, often quick inspiration. This is followed by closure of the glottis due to adduction of the vocal cords reinforced by the ventricular bands. This results in an -increase in the intrapulmonary pressure, the compressive phase, immediately preceding expiration. During the expiratory phase the vocal cords and ventricular bands are quickly separated and air is forced out with the production of characteristic cough sounds.

- The function or purpose of cough is the removal of mucus, inflammatory exudate and other material which may have originated in the air passages or foreign bodies and other materials which may have been aspirated into the tracheobronchial tree.

Cough is a complex act and its effectiveness is dependant on a number of factors in addition to the expiratory blast. Bronchologists who have had opportunity to observe the interior of the air passages during cough have been able to add enormously to our knowledge concerning this mechanism. Important among these factors are bronchial movements which are dependant upon flexibility of the air passages and ability of bronchi to elongate and increase their diameter during inspiration and to shorten and decrease their diameter during expiration. During ordinary breathing the to and fro movement of the bronchial walls unquestionably contributes much towards loosening up and moving secretions attached to the walls and probably aids ciliary function by this means. The movement of the air current during quiet respiration very probably exerts no influence on the movement of secretion unless the quantity is sufficient to almost completely obstruct a bronchus. During forced respiration there is unusual widening as well as elongation

$$
1-92
$$




\section{匃食会報一3卷}

of the bronchi during inspiration and the expiratory blast is greatly in excess of that observed in quiet breathing. During the expiratory phase there is remarkable narrowing of the bronchi due to contraction of the smooth muscles in their walls as well as forcible compression of the peripheral portions of the lung through action of the diaphragm and the chest wall.

The narrowing of the bronchus always is more at the expense of the posterior or noncartilaginous segment and is greatly accentuated in asthmatics and in cases of pulmonary emphysema. It is observed least of all in persons with pulmonary fibrosis or anthrasilicosis. This compressive action serves a useful purpose in forcing upward into larger bronchi secretions from the peripheral portion of the lungs. While respiration of a part of secretions occurs during inspiration a considerable quantity is evacuated if there are successive expiratory efforts.

The role played by bronchial movements during respiration is extremely important in the removal of secretions from the air passages and probably is not fully appreciated. Deep inspiration with forced expiration are effective since the excursus of motion of the bronchial walls is greater and this tends to move bronchial secretions and ultimately aids in their expulsion. The degree of mobility is practically negligible when breathing is quiet and shallow irrespective of its rate. This is observed in narcosis, acute pleurisy and injuries to the chest.

Another mechanism which is important in the elimination of secretions from the air passages is ciliary function. Ciliated epithelium is present in all portions of the airway except the terminal bronchioles. Under normal conditions ciliary motion and bronchial movesnents contribute in moving secretions from the smaller to the larger bronchi. Cilia often are hampered by inflammatory changes and during acute infections their activity may be greatly impaired. The presence of excessive or tenacious secretions clogs ciliary activity so that it becomes practically functionless. It is more effective in the presence of thin than in thick secretion and this should be a consideration in therapy to increase the effectiveness of cough.

A very important physiological fact, namely, the establishment of a condition of tolerance must be borne in mind in a consideration of cough. Tolerance may lessen or entirely obliterate temporarily the reflex excitability of the cough excitation areas. As cxamples may be cited the tolerance established for the O'Dwyer intubation tube when introduced into the larynx and the absence of cough following the initial paroxysm of coughing, gagging and choking following aspiration of a foreign body into the larynx or tracheobronchial tree. This has been corroborated by all bronchologists, for it is a common experience that even in the absence of local anesthesia, cough will cease following introduction of a bronchiscope into the trachea or a bronchus provided the tube is allowed to remain in contract with th: walls for a matter of seconds without touching new mucosal areas and producing reflex excitation. Whether this is due to exhaustion of the nerve endings in the mucous membrane is a question. A condition of tolerance is commonly observed in patients with bronchiectasis who have enormous quantitites of secretion. They are able to carry on without coughing for hours and when cough is initiated volitionally or by change of posture they may evacuate.three or four ounces of pus and 

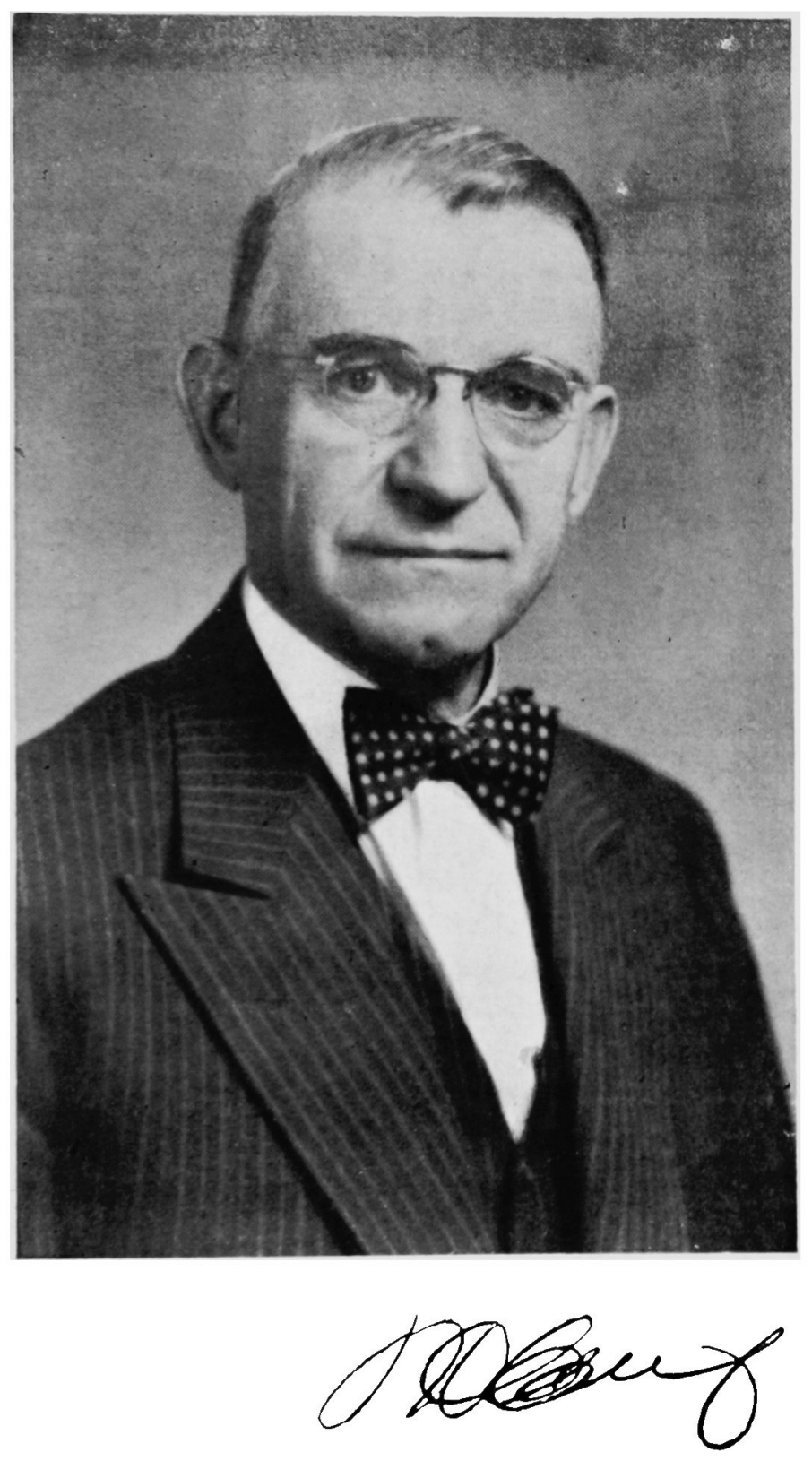


\section{氏の略歴}

フイラテルフイアのジェファーソン欧人教授

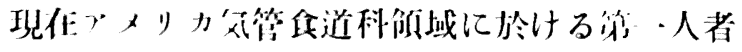

现收到学会会以

元父管任近科学公会上

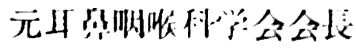

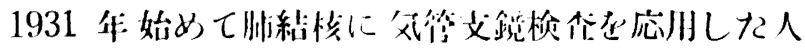

である。 
again relapse into a state of tolerance until the bronchi again fill. A similar condition has been noted in toxic conditions, anoxia, status asthmaticus, postoperative pulmonary atelectasis and drowned lung. In children one also occasionally observes a case of almost complete absence of cough reflex.

The older clinicians attached much importance to the character of the cough and this gave rise to many descriptive terms. A brassy or metallic cough was commonly associated with aneurysm of the arch of the aorta. This is due to tracheal narrowing and may also be observed in compression due to other causes and to cicatricial stenosis of the trachea, or of a bronchus. The croupy or laryngeal cough is commonly associated with conditions producing subglottic swelling. Paroxysmal cough as the name implies occurs in fits or paroxysms and is associated with certain specific respiratory infections but may occur in individuals who have an excessive amount of secretion. Cough often is referred to as dry or productive. This classification is not dependant on the patient's ability or lack of ability to expectorate after coughing, for expectoration is an accomplishment that comes from practicing. Young children and many women invariably swallow secretions after they are coughed up into the pharynx. A dry cough therefore does not necessarily mean that there is no secretion although an irrjfative cough is observed in bronchial carcinoma, often is unassociated with secretions and is truly non-productive. Not infrequently, however, individuals who have secretion in the peripheral portions of the bronchial tree are unable to cough it up as the cough is inadequate.

Since cough is a symptom one must ascertain the cause in order to treat it intelligently. Suppression of cough by administration of narcotics to inhibit the cough reflex may be beneficial at times but it is not adequate or appropriate treatment unless one is aware of the cause of the cough and that suppression is desirable.

What are the causes of cough? In the common respiratory diseases cough is a frequent symptom and there should be no difficulty in determining its cause. This applies notably to pulmonary tuberculosis, pulmonary abscess or other pulmonary diseases that can be demonstrated by routine roentgen study and physical examination. It is important however, to recognize the importance of cough as a symptom, in order that appropriate investigation may be instituted. Too often patients with carcinoma of a bronchus have appeared at the Clinic with a history of having been under medical treatment for six or more months and no diagnosis has been made. Often medication has been given to control a persistent cough which if recognized as due to bronchial irritation might have indicated the need for further bronchopulmonary investigation.

The almost universal habit of smoking, notably cigarettes has misled many persons, both patients and physicians to believe that a cough was merely a "cigarette cough" and therefore could be ignored. Cough is common among excessive smokers particularly those who smoke cigarettes. It is usually a morning cough but may be present throughout the day. It is commonly paroxysmal and is productive of tenacious secretion. There often is associated retching and gagging particularly following the first paroxysm in the morning. It is important therefore to distinguish between the cough due to certain habits and the cough due to disease of the tracheobronchial tree. Excessive smoking and chronic alcoholism produce local congestive changes in the pharynx, larynx and tracheobronehial 


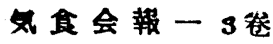

tree. These together with systemic effects, undoubtedly are responsible for the chronic inflammatory changes of the mucosa. The increased production of thick tenacious mucus which is difficult to dislogge gives rise not only to cough but frequent clearing of the throat.

Certain occupations requiring exposure to dust and fumes exert an unfavorable influence on mucous membranes of the respiratory tract. These produce chronic congestive changes, an increase in secretion and cough. In a study of patients complaining of cough it is therefore important to make inquiry into their occupation and habits.

There may be extrarespiratory causes of cough. We all are familiar with the so-called "ear cough" or "reflex aural cough" which can be provoked at will by irritating the external auditory canal. Cough may be a symptom of impacted cerumen. It is important therefore to examine the ears when investigating a case where complaint is made of cough alone. Cough may occur in eczema, local inflammation and foreign bodies in the external auditory canal.

Cough also is observed in diseases of the nose and nasal accessory sinuses. Here too it may be unassociated with any other symptom. It is important therefore in unexplained cough to investigate the nose for evidences of large turbinates, polyps, points of contact between the nasal septum and turbinates and vasomotor disturbances. The latter would probably be part of an allergic condition.

Nasal and pharyngeal obstruction is a common cause of cough particularly in children and usually is worse at night occurring after several hours of sleep. Not infrequently one observes patients with nasal disease who have been treated as cases of tuberculosis or bronchiectasis although positive findings to support this diagnosis never have been secured. In these the cough is persistent, is not controlled by the commonly employed sedatives, is associated with little sputum and usually is worse at night. Examination may reveal unsuspected disease of the sinuses or obstruction to the nasopharynx by adenoids, necessitating mouth breathing, particularly when asleep. Any condition causing nasal obstruction and mouth breathing is productive of a chronic laryngotracheitio, if continued for a sufficiently long period of time. Cough is a common symptom in this group.

Cough may be associated with the taking of food or fluid, notably thin liquids, as water. This may occur in paralysis of the larynx, either motor or sensory, esophageal stenosis, pharyngeal diverticulum, esophageal fistula communicating with the trachea or a bronchus or the presence of laryngeal disease which. interferes with the protective function of the larynx. It is important to distinguish between cause and effect when considering inflammatory conditions of the larynx as the cause of cough.

Posture at times exerts a very definite influence on the occurrence of cough. Patients with pharyngeal diverticulum often cough when assuming a prone posture. This is due to overflow of secretions of food particles into the airway. This also is observed in those who have high esophageal stenotic lesions with retention of secretions or bits of food proximal to the stenosis. In these, cough is troublesome during sleep and may be paroxysmal. Severe productive cough occurring when one assumes a prone position or a change in posture should suggest either pulmonary abscess, bronchiectasis or empyema 
with bronchopleural fistula. This is due to a change in the fluid level of secretions which, making contact with new areas of mucosa, stimulate the cough reflex.

A pedunculated tumor, foreign body or exudate in the tracheobronchial tree which on ehange of posture alters its position and make contact with new areas of mucosa will cause cough.

Allergy always must be considered as a cause of cough. It may produce generalized engorgement of the mucosa of the nose producing obstruction or similar localized changes suggesting angioneurotic edema may occur in the pharynx, larynx or tracheobronchial tree. As a rule these are unassociated with sputum.

Cough of functional origin does occur but one must be rxceedingly careful to exclude all possible organic causes before arriving at this diagnosis.

Since the potential causes of cough are innumerable how should one proceed in an investigation of this symptom? A careful history of the onset and character of the cough, the presence and appearances of sputum, the time of occurrence and associated symptoms are important. In addition one should make inquiry into the occupation and habits of the patient.

A study of the chest should be made to rule out pulmonary and mediastinal diseases. No reference has been made to cardiac disease as a cause of cough. Obviously this is an important cause and the cardiovascular system should be investigated. The more common causes of cough should be excluded first. One should then proceed with an examination of the ears, nose, mouth, throat, laryngopharynx, larynx and neck. This can be done by any physician who has a reasonable knowledge of the upper air and food passages. The inveterate smoker should be encouraged to discontinue smoking for a time and the worker in dust or fumes should make every effort to minimize exposure in the absence of any definite localizing clinical or ruentgen evidence of disease. In the absence of any demonstrable cause of cough on the basis of adecuate physical examination and roentgen study the question arises whether one should do a bronchoscopy, whether iodized oil should be instilled for lung mapping to determine if there is present bronchiectasis or whether the patient should be given a mild cough sedative and observed for a time. In the presence of unexplained radiographic shadows or localized physical signs bronchoscopy should be done particularly if the patient is an adult male. Cough without or with slight sputum is a common early symptom of bronchogenic carcinoma.

Bronchography is indicated if there is any suspicion of increased bronchopulmonary markings suggesting bronchiectasis. In the presence of a history of allergy appropriate tests should be made.

One may on occasions consider the instillation into the tracheobronchial tree of a small quantity of medicated oil to allay irritation. This is particularly useful in chronic laryngotracheitis and should be employed if the history and physical findings suggest such a diagnosis. The patient may be contented with the effects of a cough sedative or intralaryngeal instillations. The physician, however, should be interested in determining the cause of the cough as well as securing relief of this symptom.

Cough is necessary to rid the tracheobronchial tree of excessive secretions as in pulmonary abscess or bronchiectasis and in these narcotics should be used sparingly if at 
all. In carcinoma cough commonly is purposeless particularly before bronchial obstruction develops, and in these it is imperative that the symptom be recognized as an early manifestation of bronchial irritation and appropriate steps be taken to complete the diagnosis. In the postoperative case the cough "reflex" should not be suppressed. Every effort must be made to maintain adequate drainage of the tracheobronchial tree to prevent bronchial obstruction with secretions and the occurrence of postoperative pulmonary atelectasis. A similar regimen should be employed following injuries to the chest.

Much has been written concerning the use of cough mixtures in chronic cough. If cough is purposeless cough sedatives may be indicated. These should be used for brief periods however, for not only do they suppress the cough reflex but they also tend to diminish normal bron hial secretion. When cough is inadequate due either to the presence of thick tenacious secretions which adhere to bronchial mucosa and cannot be dislodged by bronchial movements or ciliary activity so-called stimulating expectorants are recommended. If these increased the fluid content of bronchial secretion so that it could be more readily coughed up there would be justification for their employment. Inhalations of carbon dioxide and oxygen increase the quantity of sputum and have been highly recommended.

In conclusion, the physician must regard cough as a symptom and while relief should be afforded the patient while the cause of cough is investigated, merely suppressing cough with a narcotic often will prove harmful. A more comprehensive knowledge of the mechanism of cough and its function is necessary to manage it successfully.

\section{気管鏡からみた浐濑}

Clerf 博士埨文の抄现

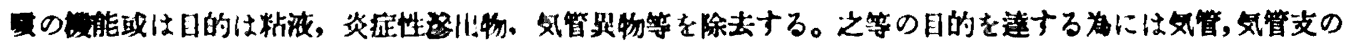
涅㽖大大いに䁌保する。

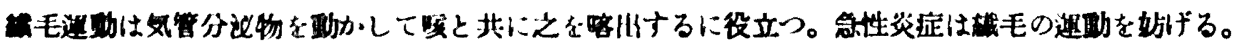

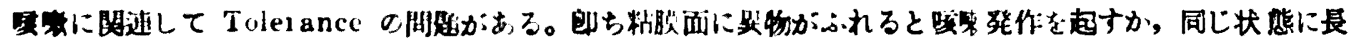

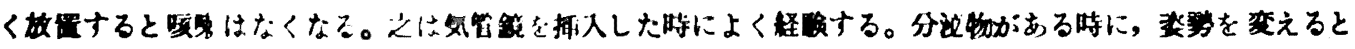

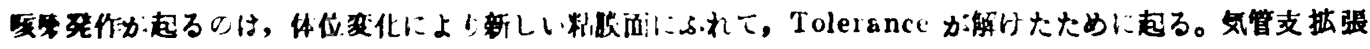

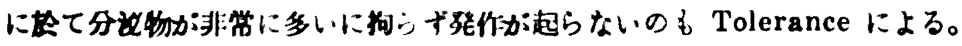

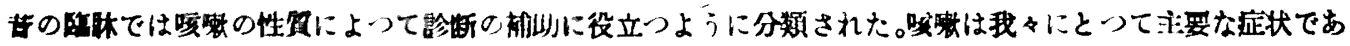

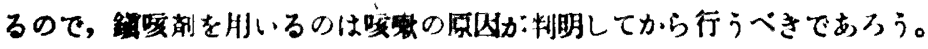

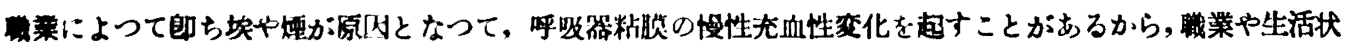
识にる注学を払う必要がある。

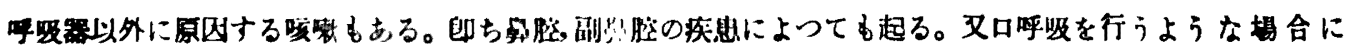

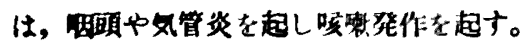

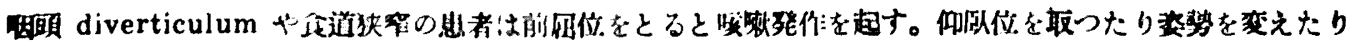

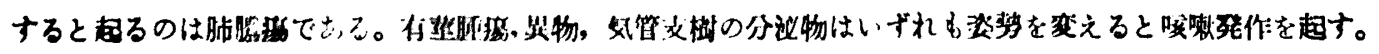

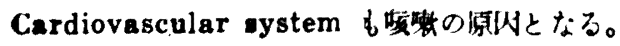

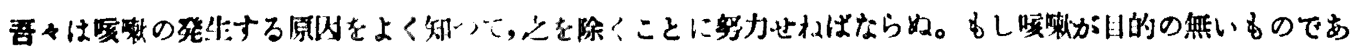

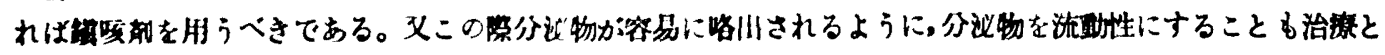
其に第竞ナぺき点である。 